\title{
Lower airway responses to rhinovirus 39 in healthy allergic and nonallergic subjects
}

\author{
D.P. Skoner*, W.J. Doyle**, J. Seroky**, M.A. Van Deusen*, P. Fireman*
}

\begin{abstract}
Lower airway responses to rhinovirus 39 in healthy allergic and nonallergic subjects. D.P. Skoner, W.J. Doyle, J. Seroky, M.A. Van Deusen, P. Fireman. CERS Journals Ltd 1996. ABSTRACT: Acute asthma is considered to be a complication of respiratory viral infections. This investigation assessed the effects of rhinovirus 39 (RV-39) infection both on the patency and responsiveness of the lower airways.

Subjects with allergic rhinitis $(A R ; n=50)$ and without $A R$ (non-AR; $n=46)$ were intranasally inoculated with $\mathrm{RV}-39$, and monitored for 8 days in an enclosed environment for changes in symptoms, signs, and airway physiology (pulmonary function, bronchial methacholine provocation). All subjects were infected postinoculation. Significant increases in nasal symptoms and secretion weights were observed, with peak effects on days 2-3. Cough was a relatively minor symptom and none of the subjects developed wheezing. Likewise, there were no significant changes in the measured functions of the lower airways. No allergy status effects were observed.

Under these experimental conditions, rhinovirus 39 infection did not produce detectable alterations in lower airway function in healthy subjects with and without allergic rhinitis.
\end{abstract}

Eur Respir J., 1996, 9, 1402-1406.
Depts of *Pediatrics and **Otolaryngology, University of Pittsburgh School of Medicine, Children's Hospital of Pittsburgh, Pittsburgh, PA, USA.

Correspondence: D.P. Skoner

Children's Hospital of Pittsburgh

3705 Fifth Avenue

Pittsburgh

PA 15213

USA

Keywords: Airway hyperreactivity, allergic rhinitis, asthma, common cold, rhinovirus, spirometry

Received: September 121995

Accepted after revision March 251996

Supported in part by the following grants: NIH AI19262, NIH MO1RR00084.
Several lines of evidence support a role for respiratory viruses in the pathogenesis of asthma, including the results of epidemiological studies [1-3]. Additional evidence is derived from studies utilizing experimental infections [4-11]. Whilst some of these studies have shown effects of respiratory viruses on lower airway function [4-6], others have not [7-11], even in asthmatic subjects [8]. Additionally, these studies suggested that allergic rhinitis (AR) may be a predisposing factor [4]. Relative limitations of these studies were the small sample sizes employed and the methodological differences in administration of viral inocula. The purpose of this study was to document the effect of experimental rhinovirus 39 (RV-39) infection on lower airway function in healthy AR and nonAR subjects. Asthmatic subjects were not included in this study, due to concern about the possible development of acute asthma in an enclosed hotel environment.

\section{Methods}

\section{Subjects}

Healthy, adult volunteers with allergic rhinitis (AR; $\mathrm{n}=50$ ) and without AR (non-AR; $n=46$ ) were identified. Allergic rhinitis was defined by the presence of a positive history, positive puncture skin test (wheal diameter $>10 \mathrm{~mm}$ ) and elevated specific serum immunoglobulin E (IgE) antibodies for inhalant allergens (concentration $>10$ IU (international units) $\cdot \mathrm{mL}^{-1}$ ). Lack of allergic rhinitis was defined by the presence of negative history, skin tests, and specific serum IgE antibodies. The majority of allergic subjects had strictly seasonal allergic rhinitis. All subjects were seronegative for neutralizing antibodies to RV-39 at baseline, as indicated by a titre of less than 2, and none had a history of asthma or other chronic diseases or active allergic or infectious symptoms at enrolment. Medication use was restricted to birth control pills. The protocol was approved by the Children's Hospital of Pittsburgh Human Rights Committee and informed consent was obtained.

The study population consisted of four cohorts of subjects who were infected with rhinovirus at different challenge sessions. This was necessitated by the physical impossibility of confining large numbers of individuals at any given challenge session. The cohorts were treated identically in every respect, including the use of the same enclosed situation, equipment, methods, rhinovirus stock, and investigative team. There were no significant cohortrelated effects on the outcome parameters of this study.

\section{Experimental protocol}

The study was conducted during months when relevant seasonal allergens were not present in the environment. Subjects were confined in individual hotel rooms for an 8 day period. Baseline spirometry, consisting of three consecutive forced expiratory manoeuvres, was performed in the morning, afternoon, and evening of the first day. The results of the test with the highest forced expiratory volume in one second (FEV1) value were recorded (FEV1, forced vital capacity (FVC), mean rate of forced expiratory flow measured between 25 and $75 \%$ of the vital capacity (FEF25-75)). In the analysis described below, the average daily value for each variable was used. 
At the end of the first day (study day 0), subjects were inoculated intranasally with a safety-tested, clinical isolate of RV-39 passaged twice in WI-38 human embryonic lung fibroblasts (provided by J. Gwaltney, Charlottesville, VA, USA). Coarse drops of the virus inoculum $(0.25$ $\mathrm{mL} \cdot$ nostril $\left.^{-1}\right)$ were delivered intranasally by pipette twice during a 30 min period (total dose $10050 \%$ tissue culture infection doses (TCID50) to seated subjects with necks hyperextended [12]. Postinoculation pulmonary function was assessed three times daily, as above, for 7 days.

\section{Illness and infection}

Daily symptoms were scored $(0=$ none to $3=$ severe $)$ and grouped as nasal (sum of scores for sneezing, rhinorrhoea, congestion), throat (sum of scores for sore throat and cough), and general (sum of scores for headache, chilliness and malaise), and secretions were weighed as described previously [12]. Daily nasal washes were cultured for RV-39 and blood was obtained at baseline and at day 23 (convalescent) to test for serum neutralizing antibodies to RV-39 [13]. Infection was defined as virus shedding on any of the postinoculation days and/or seroconversion ( $\geq$ fourfold rise in titre).

\section{Methacholine challenge}

Bronchial methacholine challenges were administered to a subset of the total subject pool. Fifty eight subjects (31 AR and 27 non-AR) received a bronchial methacholine challenge during a 2 week preconfinement, baseline period, and then again at day 7. A smaller subset of 20 subjects (11 AR and 9 non-AR) received a methacholine challenge at day 4 .

Standard methods were used [14], with sequential methacholine concentrations of 0 (saline), $0.025,0.25$, 2.5, 10 and $25 \mathrm{mg} \cdot \mathrm{mL}^{-1}$. For each, a volume of $1.0 \mathrm{~mL}$ was administered by dosimeter during five inhalations. Spirometry was assessed at 1 and $3 \mathrm{~min}$. The procedure was discontinued prematurely if the FEV1 was decreased by at least $20 \%$ from baseline on both occasions. In that case, the provocative concentration causing a $20 \%$ decrease in FEV1 (PC20) was calculated.

\section{Statistical analysis}

Viral effects on symptom scores were analysed by the two-tailed paired Student's t-test. Differences between $\mathrm{AR}$ and non-AR subjects were analysed using a repeated measures analysis of variance (ANOVA), with variance partitioned by group and study day. Longitudinal data for spirometry were analysed using a repeated measures ANOVA, with variance partitioned for day and group (allergy status) effects. For methacholine responses, the majority of subjects did not have a measurable PC20 at baseline. Therefore, within-subject differences between each post-methacholine FEV1 and the baseline FEV1 were calculated. To examine for a viral effect, the average differences were summed across challenge doses for each challenge session. The differences between preinfection and postinfection paired scores were averaged and compared to an expected difference of 0 under the null hypothesis of no effect using the two-tailed paired
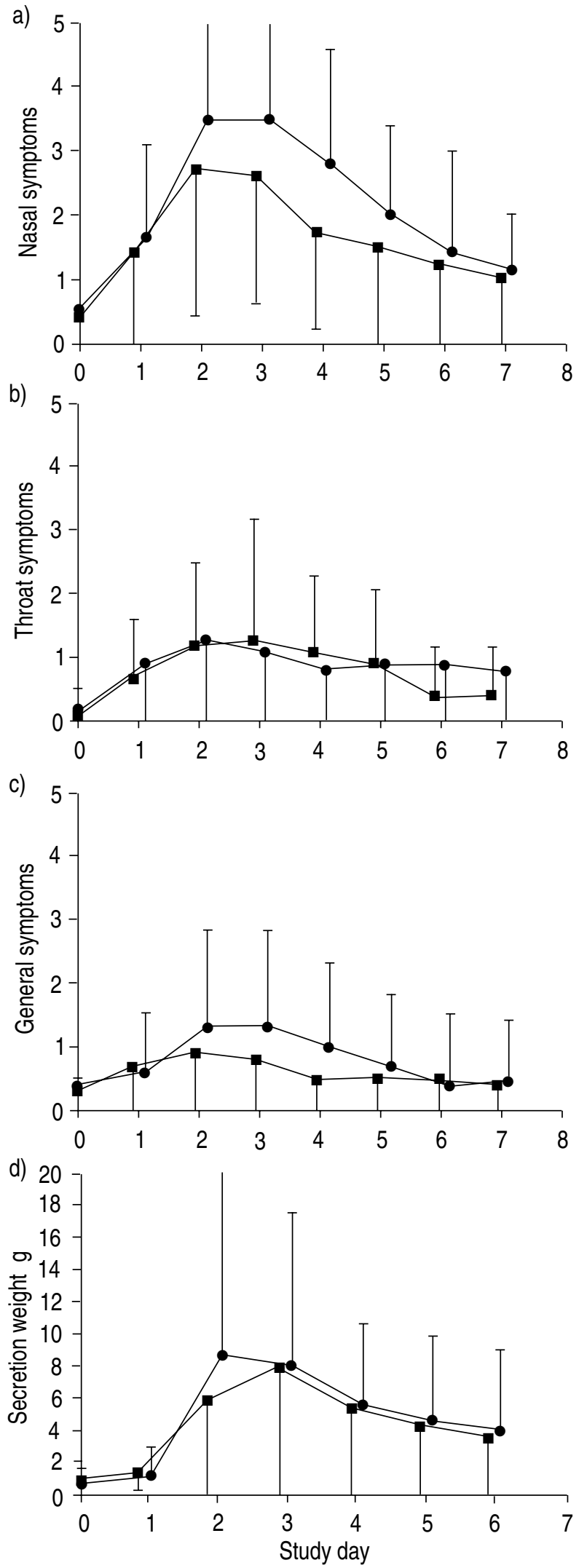

Fig. 1. - Effects of experimental rhinovirus 39 infection on: a) nasal symptoms; b) throat symptoms; c) general symptoms; and d) secretion weights, in subjects with allergic rhinitis (- - ) and without allergic rhinitis (_- ) as a function of study day. Mean values are presented; upright bars indicate $1 \mathrm{SD}$. Increases in symptoms and secretion weights with time are significant $(\mathrm{p}<0.05)$. 
t-test. Statistical significance was evaluated at a p-value of less than 0.05 .

\section{Results}

\section{Infection and illness}

All subjects were infected with RV-39 and experienced significant increases in symptoms and secretion weights $(\mathrm{p}<0.05)$, which peaked on days 2-3 (fig. 1). The "throat" summary score was dominated by sore throat, with a relatively minor contribution from cough. None of the subjects developed wheezing or other signs of lower respiratory tract disease during the study. No significant allergy status effects were observed.

\section{Pulmonary function}

Baseline pulmonary function values were greater than $90 \%$ of predicted normal values in all subjects. Additionally, the within-subject variability was $<10 \%$ for all subjects at morning, afternoon and evening testing sessions. There were no significant postinoculation changes in this parameter from baseline and no allergy status effects were observed (fig. 2).

Preinoculation, 5 of the $31 \mathrm{AR}$ subjects and 1 of the 27 non-AR subjects had a measurable PC20 to methacholine. Of these six subjects, only three, all in the AR subgroup, had a measurable $\mathrm{PC} 20$ at postinoculation day 7. The subset assessed at day 4 included two of the AR subjects and one of the non-AR subjects who had a measurable PC20 at baseline. Each of these subjects had a similar PC20 at the day 4 assessment time. A measurable $\mathrm{PC}_{20}$ at day 4 or 7 was not detected in any subject who was unresponsive at baseline. There were no significant viral effects on this parameter in AR or non-AR subjects (fig. 3).

\section{Discussion}

The results of this study showed that a large number of healthy, nonasthmatic humans did not experience detectable changes in the level of lower respiratory symptoms, pulmonary function or sensitivity of the lower airways to methacholine during an experimental RV-39 infection delivered in a highly controlled environment. Moreover, inhalant allergy was not identified as a predisposing factor. These findings were contrary to the results of most [1-3, 15, 16], but not all [17], of the earlier studies employing epidemiological techniques in asthma.

The findings were also contrary to the results of several earlier studies employing experimental designs similar to that of the current study [4-6]. In those studies, RV-16 was shown to modify bronchial hyperresponsiveness $[4,6]$, late phase allergic asthmatic reactions [4] and the lower airway inflammatory response to allergen [5]. Moreover, a modest increase in bronchial histamine responsiveness during the cold was accompanied by a modest increase in bronchial submucosal lymphocytes [18], suggesting a potential mechanism through which the RV-16 could exacerbate asthma. The number of subjects in most of these studies was small compared to the sample size of the current study. Nonetheless, the results of
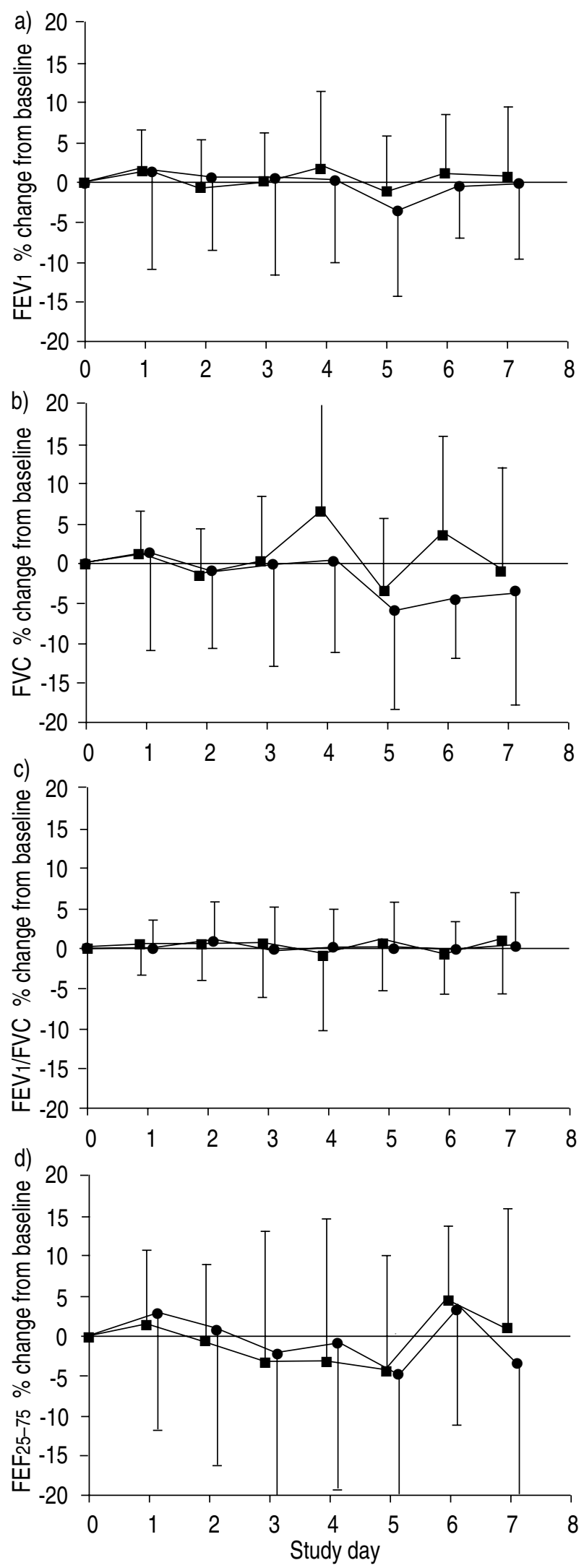

Figure 2. - Effects of experimental rhinovirus 39 infection on: a) forced expiratory volume in one second $\left(\mathrm{FEV}_{1}\right)$; b) forced vital capacity (FVC); c) FEV1/FVC ratio; and d) forced expiratory flow between 25 and $75 \%$ of FVC (FEF25-75), in subjects with allergic rhinitis (and without allergic rhinitis $(\longrightarrow$ ) as a function of study day. Mean values are presented; upright bars indicate $1 \mathrm{SD}$. 

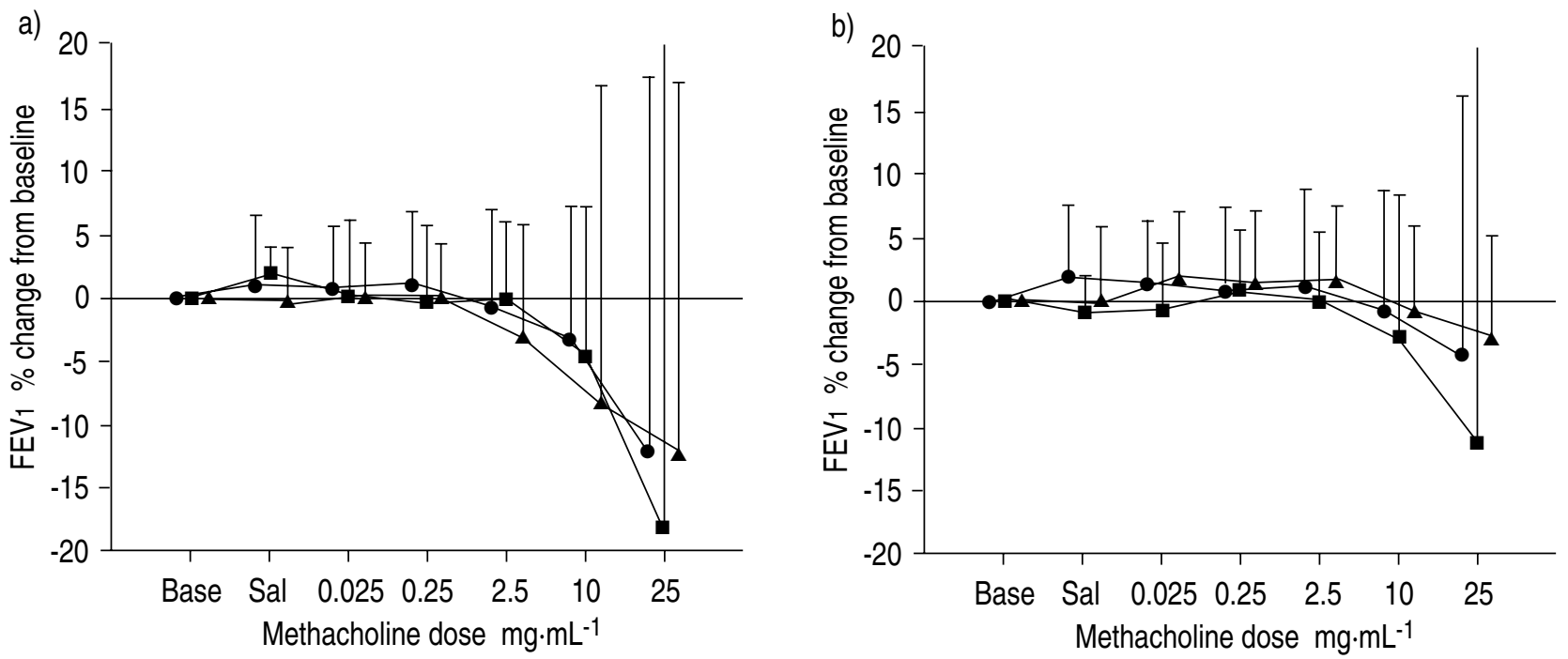

Fig. 3. - Percentage changes from baseline FEV1 as a function of methacholine dose in subjects: a) with allergic rhinitis; and b) without allergic rhinitis, at the following times in relation to rhinovirus 39 inoculation: preinoculation ( $\longrightarrow$-) ; acute-day 4 ( $\longrightarrow$ Mean values are presented; upright bars indicate 1 SD. Base: baseline; Sal: saline; FEV1: forced expiratory volume in one second.

the current study were surprising, since the enhanced leucocyte histamine release observed in conjunction with RV16-induced lower airway changes [4] was also observed in the AR subgroup of these same RV-39 infected subjects [19].

In contrast, many investigators have failed to find evidence to support a causal relationship between rhinoviruses and asthma. Indeed, none of the viruses delivered in experimental settings has been reported to trigger acute asthma or alter routine spirometric parameters, even when the study population included subjects with AR and/or asthma, and virus was cultured from the lower airways [7-11]. Similarly, conflicting results have also been reported for studies employing experimental influenza virus infections. In those studies, which also included small sample sizes, bronchial reactivity was increased in nonasthmatic $\mathrm{AR}$ and non-AR patients with natural infections [20], and in experimentally-infected asthmatic [21] and non-asthmatic [22] individuals. In contrast, studies conducted in our laboratory utilizing large numbers of subjects failed to detect an effect of experimental influenza A virus infections on the lower airways of healthy $\mathrm{AR}$ and non-AR subjects [23].

Collectively, the results of the above studies suggest that critical host, viral, environmental or experimental factors may have operated as variables in modifying outcome. One likely source of variability is the experimental model itself. Whilst such models can provide valuable information which may not be available in studies of natural colds, including the timing of virus inoculation and the precise onset and duration of symptoms and signs of infection, they also introduce methodological variability.

Specifically, the inoculation method could potentially influence the ability of viruses to affect the lower airways. Indeed, several inoculation methods have been described. These include delivery on one [10, 21-23] versus two consecutive $[46,18]$ days; and nasal drop instillation with $[4,5,18,22]$ or without $[10,21,23]$ the addition of nasal spray from an atomizer and nasal inhalation from a nebulizer [6]. Unfortunately, none of these methods, some of which have been designed to enhance lower airway involvement, has been tested for comparability or validity in controlled settings.

Other factors which may be important in determining the extent of lower airway involvement during experimental respiratory virus infections [24, 25] include the following: 1) virus and even strain specificity; 2) confinement during the acute stage of the infection; 3) the frequency of assessment of outcome parameters; 4) the type of agonist used to assess lower airway hyperresponsiveness (histamine versus methacholine); 5) timing of delivery of the acute bronchoprovocation test; and 6) whether or not the subject pool includes-predisposed subgroups.

Predisposed subgroups may include AR patients, in whom effects have extended both to the lower airways [4] and the systemic immune response [26]. Seasonal allergen priming could also potentially alter viral responses, but studies reporting both positive and negative results utilized AR subjects who were asymptomatic [4, 23]. The overall prevalence of airway hyperrepsonsiveness to methacholine in nonasthmatic patients with AR is generally considered to be about twice as high as that reported in the current study (16\%). However, the reported frequencies have been quite variable, ranging from $10-15$ to $75 \%$ [27, 28]. A low percentage in the current study may reflect the asymptomatic state of the AR subjects at the time of entry. Seasonal allergen exposure is known to heighten the degree of nonspecific bronchial reactivity [29].

In addition to the above factors, the severity of infection may also be a governing factor. The subjects of the current study were infected and experienced a significant degree of upper airway symptomatology and pathophysiology [12], in the absence of detectable changes in lower airway function. However, it is possible that a more severe infection would have affected the lower airways. Another potential factor is the sensitivity of each of the methods used to assess outcome. In the current study, it is possible that the use of specific airway conductance ( $\mathrm{s} G \mathrm{aw}$ ) or challenge with higher doses of methacholine would have produced positive results. Use of the latter test in a recent study showed that RV-16 caused a prolonged excessive airway narrowing in asthmatic subjects in vivo [6]. 
The implications of this study for normal and asymptomatic allergic humans are clear. These groups are highly unlikely to develop measurable or clinically apparent changes in lower airway function during infection with rhinovirus 39 . In contrast, the study provides no information about the implications for humans with symptomatic allergy or chronic lower respiratory tract diseases like asthma. The contrast between the results of this and earlier studies highlights the need to exercise caution when formulating experimental design and when assessing the clinical relevance of the results of investigations which employ experimental infections.

Obviously, asthmatic patients should be the ultimate target population of future studies of experimental rhinovirus infections. Our laboratories are now planning to conduct such studies, since the approach appears safe. We are also planning to conduct studies utilizing allergen-primed allergic subjects, who may be predisposed to develop lower airway effects following a rhinovirus challenge. Indeed, there may be a significant viral-allergen interaction in generating the lower airway inflammatory response [5].

\footnotetext{
Acknowledgements: The authors thank J. Gwaltney, for providing valuable expertise and laboratory resources for monitoring the viral infections, J.D. Swarts and E. Tanner, for technical assistance, C. Wagner and S. Cirota for secretarial assistance, and the administrative and nursing staff of the Centers for Asthma and Allergic Diseases and General Clinical Research of the Children's Hospital of Pittsburgh for their cooperation and assistance.
}

\section{References}

1. Beasley R, Coleman ED, Hermon Y, Holst PE, O'Donnell TV, Tobias M. Viral respiratory tract infection and exacerbations of asthma in adult patients. Thorax 1988; 43: 679-683.

2. Nicholson KG, Kent J, Ireland DC. Respiratory viruses and exacerbations of asthma in adults. BMJ 1993; 307: 982-986.

3. Minor TE, Dick EC, Baker JW, Ouellette JJ, Cohen M, Reed CE. Rhinovirus and influenza type A infections as precipitants of asthma. Am Rev Respir Dis 1996; 113: 149-153.

4. Lemanske RF, Dick EC, Swenson CA, Vrtis RF, Busse WW. Rhinovirus upper respiratory infection increases airway hyperreactivity and late asthmatic reactions. $J$ Clin Invest 1989; 83: 1-10.

5. Calhoun WJ, Dick EC, Schwartz LB, Busse WW. A common cold virus, rhinovirus 16 , potentiates airway inflammation after segmental antigen bronchoprovocation in allergic subjects. J Clin Invest 1994; 94: 2200-2208.

6. Cheung D, Dick EC, Timmers MC, de Klerk EPA, Spaan WJM, Sterk PJ. Rhinovirus inhalation causes prolonged excessive airway narrowing in asthmatic subjects in vivo. Am J Resp Crit Care Med 1996; 152: 1490-1496.

7. Halperin SA, Eggleston PA, Hendley JO, Suratt PM, Groschel DHM, Gwaltney JM. Pathogenesis of lower respiratory tract symptoms in experimental rhinovirus infection. Am Rev Respir Dis 1983; 126: 806-810.

8. Halperin SA, Eggleston PA, Beasley P, et al. Exacerbations of asthma in adults during experimental rhinovirus infection. Am Rev Respir Dis 1985; 132: 976-980.

9. Summers QA, Higgins PG, Barrow IG, Tyrrell DA, Holgate ST. Bronchial reactivity to histamine and bradykinin is unchanged after rhinovirus infection in normal subjects. Eur Respir J 1992; 5(3): 313-317.
10. Bush RK, Busse W, Flaherty D, Warshauer DM, Dick EC, Reed CE. Effects of experimental rhinovirus 16 infection on airways and leukocyte function in normal subjects. J Allergy Clin Immunol 1978; 61(2): 80-87.

11. Blair HT, Greenberg SB, Stevenson PM, Bilunos PA, Couch RB. Effects of rhinovirus infection on pulmonary function of healthy human volunteers. Am Rev Respir Dis 1976; 114: 95-102.

12. Doyle WJ, Skoner DP, Fireman P, et al. Rhinovirus 39 infection in allergic and nonallergic subjects. $J$ Allergy Clin Immunol 1992; 89: 968-978.

13. Gwaltney JM Jr. Rhinovirus. In: Mandell GL, Douglas RG JR, Bennett JE, eds. Principles and Practice of Infectious Disease. New York, John Wiley and Sons, 1979; pp.1124-1134.

14. Chai H, Farr RS, Froehlich LA, et al. Standardization of bronchial inhalation challenge procedures. J Allergy Clin Immunol 1975; 56: 323-327.

15. Cate TR, Roberts JS, Russ MA, Pierce JA. Effects of common colds on pulmonary function. Am Rev Respir Dis 1973; 108: 858-865.

16. Aquilina AT, Hall WJ, Douglas RG, Utell MJ. Airway reactivity in subjects with viral upper respiratory tract infections: the effects of exercise and cold air. Am Rev Respir Dis 1980; 122: 3-10.

17. Sokhandan M, McFadden ER Jr, Huang YT, Mazanec MB. The contribution of respiratory viruses to severe exacerbations of asthma in adults. Chest 1995; 107: 1570-1575.

18. Fraenkel DJ, Bardin PG, Sanderson G, Lampe F, Johnston SL, Holgate ST. Lower airways inflammation during rhinovirus colds in normal and in asthmatic subjects. Am J Respir Crit Care Med 1995; 151: 879-886.

19. Skoner DP, Doyle WJ, Tanner EP, Kiss J, Fireman P. Effect of rhinovirus 39 (RV-39) infection on immune and inflammatory parameters in allergic and nonallergic subjects. Clin Exper Allergy 1995; 25: 561-567.

20. Little JW, Hall WJ, Douglas RG, Jr, Mudholkar GS, Speers DM, Patel K. Airway hyperreactivity and peripheral airway dysfunction in influenza infection. Am Rev Respir Dis 1978; 118: 295-303.

21. Laitinen LA, Kava T. Bronchial reactivity following uncomplicated influenza A infection in healthy subjects and in asthmatic patients. Eur J Respir Dis 1980; 106: 51-58.

22. Laitinen LA, Elkin RB, Empey DW, Jacobs L, Mills J, Nadel JA. Bronchial hyperresponsiveness in normal subjects during attenuated influenza virus infection. Am Rev Respir Dis 1991; 143: 358-361.

23. Skoner DP, Doyle WJ, Seroky J, Fireman P. Lower airway responses to influenza $\mathrm{A}$ virus in healthy allergic and nonallergic subjects. Am J Respir Crit Care Med 1996; in press.

24. Bardin PG, Johnston SL, Pattemore PK. Viruses as precipitants of asthma symptoms. II. Physiology and mechanisms. Clin Exp Allergy 1992; 22: 809-822.

25. Sterk PJ. Virus-induced airway hyperresponsiveness in man. Eur Respir J 1993; 6: 894-902.

26. Skoner DP, Whiteside TL, Wilson JW, Doyle WJ, Herberman RB, Fireman P. Effect of rhinovirus 39 (RV39) infection on cellular immune parameters in allergic and nonallergic subjects. J Allergy Clin Immunol 1993; 92: 732-743.

27. Cockcroft DW. Nonallergic airway responsiveness. $J$ Allergy Clin Immunol 1988; 81: 111-118.

28. Robalo-Cordeiro AJA, Pinto-Mendes JA, Carvalho JHP, et al. Bronchial hyperreactivity in allergic upper respiratory disease. In: Herzog H, Perruchoud AP, eds. Progress in Respiratory Research. Asthma and Bronchial Hyperreactivity. Vol. 19. Basel, Karger, 1985; pp. 285-289.

29. Madonini E, Briatico-Vangosa G, Pappacoda A, et al. Seasonal increase of bronchial reactivity in allergic rhinitis. J Allergy Clin Immunol 1987; 79: 358-363. 